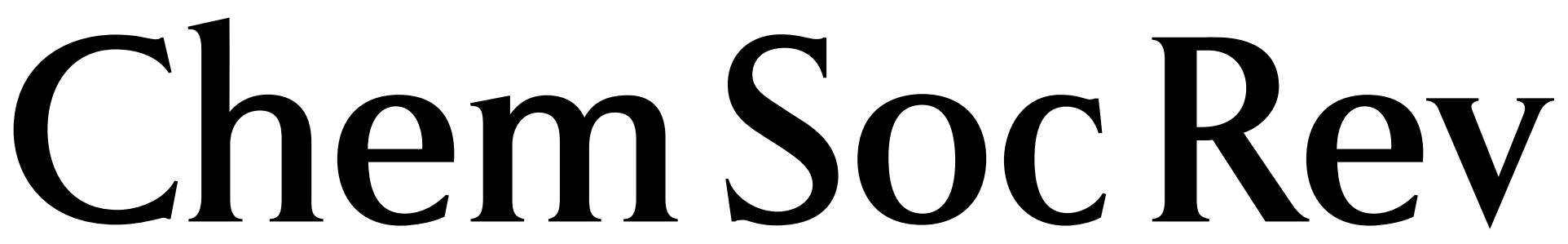

Chemical Society Reviews

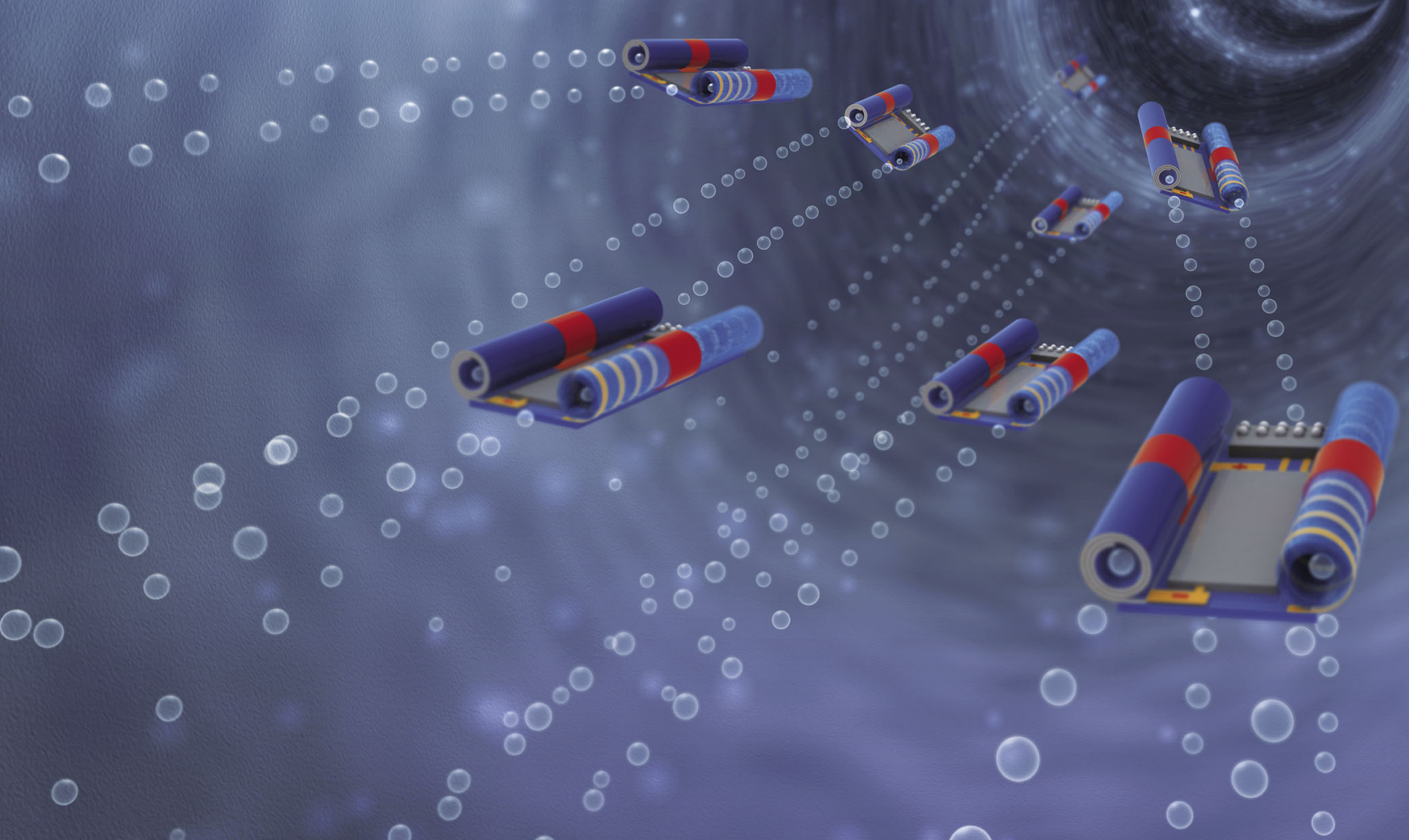


Cite this: Chem. Soc. Rev., 2011, 40, 2109-2119

\title{
Rolled-up nanotech on polymers: from basic perception to self-propelled catalytic microengines
}

\author{
Yongfeng Mei, ${ }^{\star a b}$ Alexander A. Solovev, ${ }^{b}$ Samuel Sanchez $^{b}$ and \\ Oliver G. Schmidt $* b c$ \\ Received 13th August 2010 \\ DOI: $10.1039 / \mathrm{c0cs00078g}$
}

In this tutorial review we describe the recent progress on catalytic microtubular engines fabricated by rolled-up nanotech on polymers. We summarize the technical aspects of the technology and the basic principles that cause the catalytic microengines to self-propel in fuel solutions. The control over speed, directionality and interactions of the microengines to perform tasks such as cargo transportation is also discussed. We compare this technology to other fabrication techniques of catalytic micro-/nanomotors and outline challenges and opportunities for such engines in future studies. Since rolled-up nanotech on polymers can easily integrate almost any type of inorganic material, huge potential and advanced performance such as high speed, cargo delivery, motion control, and dynamic assembly are foreseen - ultimately promising a practical way to construct versatile and intelligent catalytic tubular microrobots.

\section{Introduction}

A jet engine is a reaction engine that discharges a fast moving jet of fluid or gas to generate thrust by jet propulsion

${ }^{a}$ Department of Materials Science, Fudan University, Shanghai, 200433, People's Republic of China.

E-mail: yongfeng.mei@gmail.com

${ }^{b}$ Institute for Integrative Nanosciences, IFW Dresden, Helmholtzstr. 20,D-01069 Dresden, Germany.E-mail: o.schmidt@ifw-dresden.de ${ }^{c}$ Material Systems for Nanoelectronics, Chemnitz University of Technology, Reichenhainer Str. 70, D-09107 Chemnitz, Germany consistent with classic Newton's laws of motion. ${ }^{1}$ However, Purcell explained in 1977 that inertia plays no role if objects move at very low Reynolds numbers because the coasting time is very short as well as the coasting distance. ${ }^{2}$ It is known that a high fluid viscosity and/or a small object generate low Reynolds numbers, where friction dominates over inertia of mass. Furthermore, Brownian motion becomes comparable and can even dominate over directional motion at small scales. One typical approach to fabricate autonomous directional motility at low Reynolds numbers is to convert the chemical

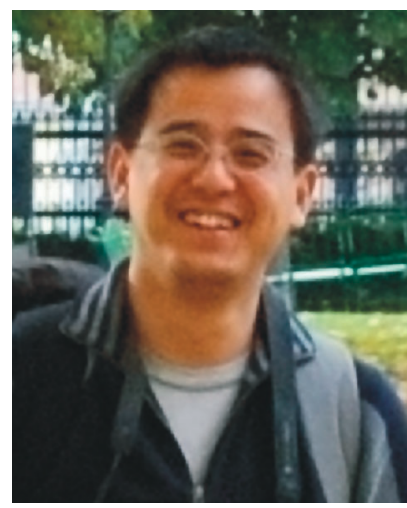

Yongfeng Mei
Yongfeng Mei received his PhD at City University of Hong Kong in 2005. Thereafter, he joined the Molecular Beam Epitaxy Group as a post-doctoral researcher in the Max Planck Institute for Solid State Research. The topic was on wrinkled-up micro/nanochannels for nanofluidics and optics. In 2007, he moved to IFW Dresden as a staff scientist and a group leader in the Institute for Integrative Nanosciences. His group mainly focused on Nanomembranes Technology for Applications of Biology and Chemistry (Nano-ABC). In 2010, he joined the Department of Materials Science, Fudan University, China, as a full professor in materials chemistry and physics.

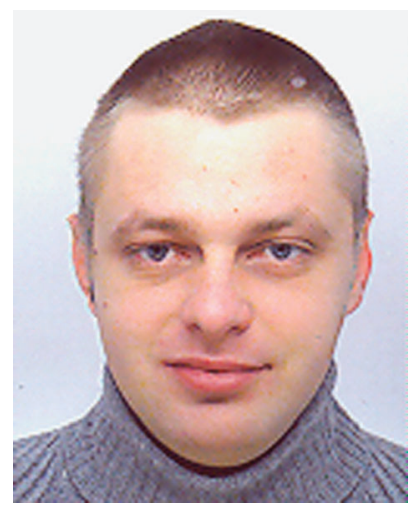

Alexander A. Solovev
Alexander A. Solovev graduated with Master of Science in Engineering Physics from Walther Schottky Institute, Physics Department at Technical University of Munich, Germany, in 2004. During 2003-2004 he was a visiting student at Princeton University and Columbia University in the City of New York, USA. He joined the Molecular Beam Epitaxy Group at the Max Planck Institute for Solid State Research at Stutgart, Germany, in 2005. He is currently a PhD candidate working with Prof. O. G. Schmidt at the Leibniz Institute for Solid State Physics and Materials Research, Dresden, Germany. His research is focused on the development and applications of micro- and nanoscale engines powered by catalytic reactions. 
(a)

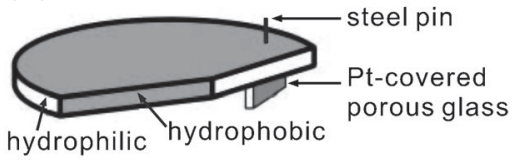

(b)

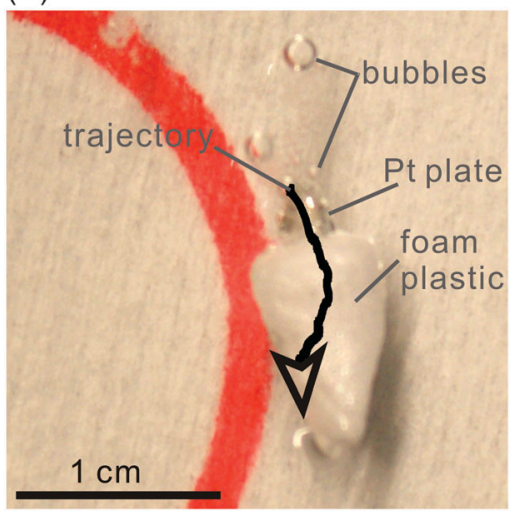

Fig. 1 (a) Schematic of a self-propelling object (a thin plate with circa 1-2 $\mathrm{mm}$ thickness and $9 \mathrm{~mm}$ diameter). Reprinted with permission from ref. 3. (b) A simple and hand-made catalytic "boat". A Pt plate was inserted into a foam plastic and placed onto the fuel $\left(\mathrm{H}_{2} \mathrm{O}_{2}\right.$ aqueous solution) surface.

energy taken from the accessible environment into mechanical energy. Whitesides et al. have illustrated this approach with an object on the millimetre scale. ${ }^{3}$ As shown in Fig. 1a, a thin plate was fabricated from polydimethylsiloxane (PDMS) into the desired shape. A piece of porous glass filter covered with platinum (Pt) was mounted on the PDMS piece with a stainless steel pin. Pt decomposes the fuel hydrogen peroxide $\left(\mathrm{H}_{2} \mathrm{O}_{2}\right)$ into water $\left(\mathrm{H}_{2} \mathrm{O}\right)$ and oxygen gas $\left(\mathrm{O}_{2}\right)$. The oxygen bubbles recoil the object and cause a movement of the plate. A simple and hand-made catalytic "boat" is also shown in
Fig. 1b. The Pt plate was inserted into a foam plastic and placed onto the fuel $\left(\mathrm{H}_{2} \mathrm{O}_{2}\right.$ aqueous solution) surface. Gaseous oxygen bubbles formed immediately and directly propelled the boat at the liquid surface. In 2004, two research groups (one from Pennsylvania State University ${ }^{4}$ and the other from University of Toronto) $)^{5}$ independently found that bimetal nanorods can serve as catalytic nanomotors in a $\mathrm{H}_{2} \mathrm{O}_{2}$ solution. Interestingly, movements in opposite directions were found and explained by interfacial tension gradients, ${ }^{4}$ gas bubble propulsion, ${ }^{5}$ and self-electrophoresis mechanisms. ${ }^{6}$ Since then, substantial effort has been dedicated to develop and understand catalytic autonomous motions involving compositions and shapes of catalysts, types of chemical fuels, cargo functionality, magnetic remote control, and on-chip integration. ${ }^{?}$

In this field, three issues currently attract great attention. One is understanding the driving principles of the motion, since there are several explanations for the observed phenomena. Except for the interfacial tension gradient and the bubble propulsion, other mechanisms have been suggested in several reviews. ${ }^{8-14}$ Still, it seems that there is no general solution which can clarify or justify the origin of catalytic motions at small scale. At least, a unified understanding is still missing at the current stage. The second fascinating part is the control of catalytic motion. ${ }^{13,15}$ Magnetic remote guidance is a simple and efficient way to direct cargo transportation. ${ }^{13,16}$ Recently, Wang and Manesh have developed different strategies to control the motion of catalytic nanomotors like thermally driven acceleration, electrochemical switching, and chemical stimuli (including control of the fuel concentration). ${ }^{15}$ In the meantime, Sen and co-workers have also demonstrated new functionality with catalytic nanomotors, for instance, chemotaxis and phototaxis. ${ }^{13}$ Further improvement of a precise temporal and/or spatial control could enable catalytic nanomotors to dynamically self-assemble for

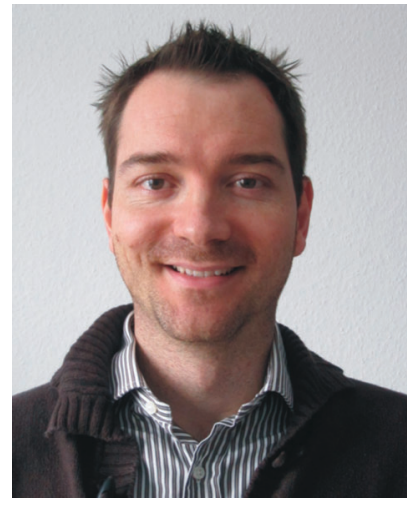

Samuel Sanchez
Samuel Sanchez received his $\mathrm{PhD}$ at the Autonomous University of Barcelona, Spain, in 2008. He studied and developed new composite materials for the construction of electrochemical biosensors based on soft polymers, carbon nanotubes, and metallic nanoparticles. After one year as assistant professor at the Autonomous University of Barcelona, he joined the ICYS at the International Research Center for Materials Nanoarchitectonics (MANA), National Institute for Materials Science, Japan, in February 2009 in a tenure track position. In May 2010 he joined the Institute for Integrative Nanoscience at the Leibniz Institute in Dresden where currently he is leading a group working on NanoBiochemical applications of nanomembranes.

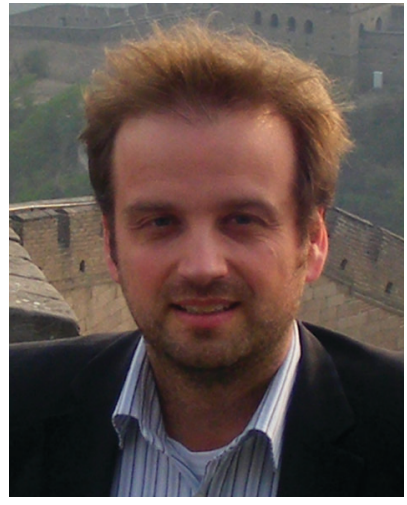

Oliver G. Schmidt
Professor Oliver G. Schmidt carried out his PhD thesis on self-assembled SiGeC nanostructures at the Max-Planck Institute for Solid State Research in Stuttgart, Germany, from 1996 to 1999. At the same institute he continued research on straindriven nanostructures and received his Habilitation in 2003. From 2003 to 2007 he supervised a research group in Stuttgart, concentrating on self-assembled quantum dots and rolled-up nanotech. Since 2007 he is a Director at the IFW Dresden, Germany, and at the same time holds a full professorship in Electrical Engineering at the Chemnitz University of Technology. He was awarded various prizes including the Philip Morris Research Award in 2002 and the Carus Medal in 2006. Since 2006 he is co-editor of the journal Nanoscale Research Letters. His research spans from nanophotonics to cellular biophysics and nanorobotics. 
patterning, sensing their environment and communicating with each other. Such artificial motors could also learn from nature and help understand biological motion at small scale. ${ }^{17}$ This is then the third issue in catalytic micro- and nanomotors: the development of, for example, new biocompatible fuels, environmental sensing, and memory effects of "intelligent motors" 16 or "dream machines", 9 which are expected to compete with nature's biomotors in the not-so-distant future. ${ }^{17}$

Recently, a new type of catalytic tubular microengine has been developed and fabricated by rolled-up nanotechnology ${ }^{7,18,20}$ and templated electrodeposition. ${ }^{19}$ Such tubular engines offer several advantages, like easy motion control, integration of various functions, scalable size in diameter and length, straight trajectories, etc. ${ }^{7,16,18}$ This tutorial article describes the progress of catalytic tubular microengines in view of fabrication, basic principles, speed issues, motion control, cargo transportation and assembly, and dynamic interaction. The tutorial concentrates on rolled-up tubular microengines and compares them to other catalytic motors throughout the text. Section 2 describes the fabrication process of rolled-up nanotech on polymers. In Section 3, the basic working principle of our catalytic tubular microengines is clarified. In Section 4, we discuss speed issues (including fundamental limitations) of our engines and compare these with other motors. Section 5 focuses on trajectories and motion control. Cargo transportation and assembly is discussed in Section 6 together with pumping effects. Section 7 highlights our recent progress on catalytic microtubular "water striders". The article is finalized with a summary and outlook of future directions in tubular microengines as well as other catalytic motors at the micro- and nanoscale.

\section{Rolled-up nanotech on polymers}

Strain engineering offers a great opportunity to deterministically rearrange nanomembranes into three dimensional micro/ nanostructures ${ }^{21}$ including tubes, ${ }^{18,20}$ helices, ${ }^{22}$ and wrinkles. ${ }^{23}$ In principle, a wide range of possible materials can be used for this method employing appropriate deposition techniques and a selective etchant which removes the underlying sacrificial layer. Incorporating an engineered strain gradient in the deposited films, practically any thin solid film can roll up into a micro/nanotube at almost any designated position once released from its substrate. However, the selective underetching often not only removes the underlying sacrificial layer but also in many cases dissolves the functional layer which forms the rolled-up tube. Experimentally, our experience is that the etching rate of a selected etchant to the sacrificial layer should be 1000 times higher than the dissolving rate of the functional layer. Here, we adopt a polymer thin film as a sacrificial layer since acetone can etch polymers over almost any inorganic materials with practically $100 \%$ selectivity. ${ }^{18} \mathrm{~A}$ prestressed inorganic nanomembrane deposited at low temperatures onto a photoresist layer is released from the substrate by removing the sacrificial layer with acetone, and it rolls-up into a micro-/ nanotube as shown in Fig. 2a. Examples of rolled-up tubes are shown in Fig. 2b. For accurate positioning and tube integration on a single chip, we apply a tilted deposition method with e-beam and thermal evaporation. In this case, a narrow gap remains open after deposition at the far end of the pattern due to the ballistic shadow effect. ${ }^{18}$ The opposite side is well-sealed and holds/fixes the rolled-up tubes once formed. As shown in Fig. 2c, tubes of well-defined length and geometry can be arranged into large periodic arrays as demonstrated for $\mathrm{SiO} / \mathrm{SiO}_{2}$ microtubes on a $\mathrm{Si}$ substrate. Such arrays can be mass-produced on almost any flat substrate like glass or plastic.

The tube diameter can be tuned by changing the thickness and the built-in strain of the functional layer as shown in Fig. 2d. The grey lines represent simplified calculated data. The smallest tube we obtained by using rolled-up nanotech on polymers is $\sim 115 \mathrm{~nm}$ in radius (left inset), which rolled-up from a one-nanometre-thick $\mathrm{Al}_{2} \mathrm{O}_{3}$ film grown by atomic layer deposition (ALD). For diameters between one to several tens of microns, rolled-up nanotech can produce microtubes with well-defined geometry as shown in the right inset of Fig. $2 d$ as well as Fig. 2c. Compared to thin solid films grown by molecular beam epitaxy, the deposited films by the e-beam or thermal evaporation method and ALD are normally amorphous or polycrystalline. As a result, mechanical stability is often limited and critical point drying is necessary for the fabrication process to avoid any tube collapsing due to surface tension of the liquid etchants. Also, the strain in molecular beam expitaxy (MBE)-grown films can be well tuned by lattice mismatch. $^{24}$ In the amorphous or polycrystalline films, the strain is much harder to access, ${ }^{18}$ but simple design rules can be defined and followed. Initial experiments can clarify the strain state of single layers under various parameters. For a suitable bilayer deposition the parameters from the initial experiments are taken and combined to maximize the strain difference. This easy approach can help in many cases to create rolled-up tubes with predictable diameters but for certain material combinations fails since bilayer film deposition represents a complex coating scenario with many variable interdependencies.

Still, rolled-up nanotech on polymers can generate micro/ nanotubes made of almost any inorganic material (Fig. 2b) on almost any flat substrate, ${ }^{18}$ and provides unique flexibility to explore even more functionalities based on shaped hybrid nanomembranes. For catalytic motion, the flexibility offers various possibilities to integrate control or functional components into tubular microengines.

\section{Technical note}

The following process steps are required to create rolled-up microengines. A photoresist layer with a thickness of $1-2 \mu \mathrm{m}$ is spin coated on a $\mathrm{Si}$ substrate applying e.g. conventional ARP-3510 from Allresist GmbH. The photoresist film is then patterned by photolithography. Afterwards, metals (e.g. Pt, $\mathrm{Ti}, \mathrm{Cr}, \mathrm{Au}$, and $\mathrm{Fe}$ ) are deposited by e-beam evaporation under high vacuum conditions $\left(<10^{-4} \mathrm{~Pa}\right)$. After selectively removing the sacrificial photoresist in appropriate solvents (acetone, ethanol, and isopropanol), critical point drying (CPD 030 Critical Point Dryer from Bal-Tec AG) is applied to dry the rolled-up nanomembranes without structural collapse. A droplet of isopropanol containing microtubes is then placed on the stage under a microscope after adding 
(a)

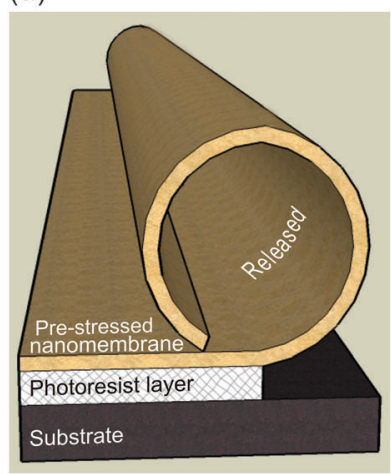

(c)

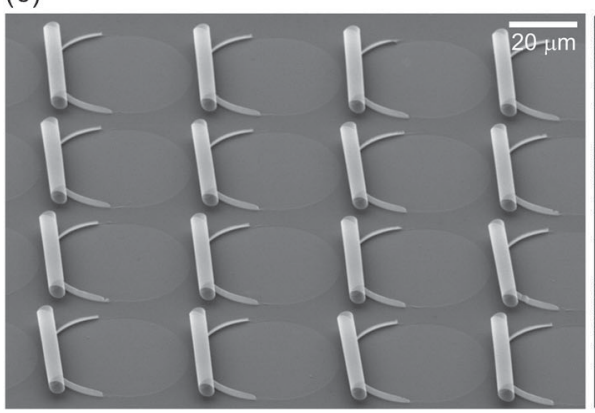

(b)

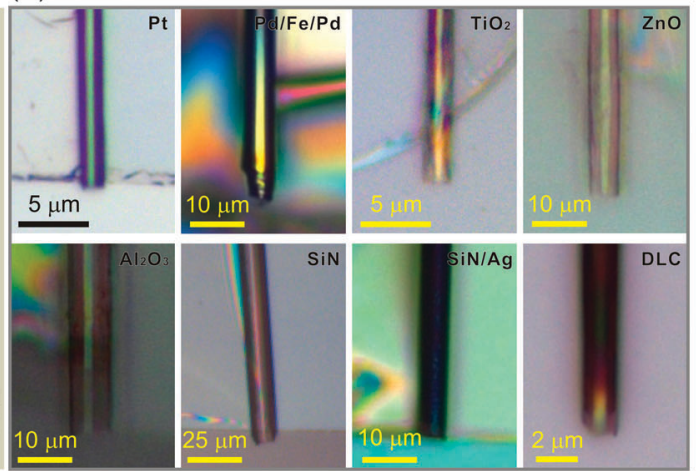

(d)

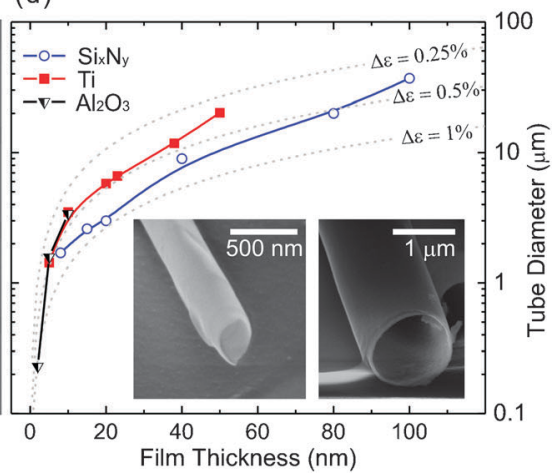

Fig. 2 (a) Schematic diagram illustrating the roll-up process of a nanomembrane into a tube on photoresist; optical images of rolled-up nanomembranes made out of (b) $\mathrm{Pt}, \mathrm{Pd} / \mathrm{Fe} / \mathrm{Pd}, \mathrm{TiO}_{2}, \mathrm{ZnO}, \mathrm{Al}_{2} \mathrm{O}_{3}, \mathrm{Si}_{x} \mathrm{~N}_{y}, \mathrm{Si}_{x} \mathrm{~N}_{y} / \mathrm{Ag}$, and DLC; (c) SEM image of an array of rolled-up SiO/SiO${ }_{2}$ nanomembranes; (d) tube diameters as a function of film thickness for various materials: $\mathrm{Si}_{x} \mathrm{~N}_{y}$ (blue open circles), Ti (red filled squares), and $\mathrm{Al}_{2} \mathrm{O}_{3}$ (black half-filled triangles). Left inset shows an $\mathrm{SEM}$ image of an $\mathrm{Al}_{2} \mathrm{O}_{3}$ tube with a diameter of $\sim 230 \mathrm{~nm}$. Right inset displays an SEM image of a $\mathrm{Si}_{x} \mathrm{~N}_{y}$ microtube. Reprinted with permission from ref. 18.

$\mathrm{H}_{2} \mathrm{O}_{2}$ aqueous solution at various concentrations. Video microscopy is recorded at speeds from 50 to 600 frames per second with a high speed camera (Photonic Science Limited) attached to an optical microscope (Zeiss Axiotech vario). The catalytic inside wall of the tubes (consisting of e.g. Pt) decomposes $\mathrm{H}_{2} \mathrm{O}_{2}$ into water and $\mathrm{O}_{2}$. The produced $\mathrm{O}_{2}$ accumulates inside the tube and forms gas bubbles which are ejected from one tube end and cause the propulsion of the microengine in the opposite direction.

\section{Principle of catalytic tubular microengines}

It is well known that catalysts like $\mathrm{Pt}$ or $\mathrm{Ag}$ decompose $\mathrm{H}_{2} \mathrm{O}_{2}$ into $\mathrm{O}_{2}$ and water, which is the origin of the driving force in catalytic micro- and nanomotors in a fuel $\left(e . g . \mathrm{H}_{2} \mathrm{O}_{2}\right)$ solution. ${ }^{3-6}$ However, such a catalytic reaction involves many subsequent processes, thus rendering a definite interpretation of the mechanisms difficult. For example, if there is a concentration gradient of the solute or the reaction products created during the reaction around the motors, mechanisms such as diffusiophoresis or osmophoresis ${ }^{8}$ could prevail. Furthermore, the gradient of gaseous $\mathrm{O}_{2}$ in a bimetal (e.g. $\mathrm{Pt}-\mathrm{Au}$ ) nanorod would also result in an asymmetric interfacial tension gradient, which induces a slip velocity at the particlefluid interface (denoted as interfacial tension gradient mechanism). ${ }^{8}$ Another mechanism, self-electrophoresis, originates from a redox reaction occurring in the catalytic segment, where the produced protons at one end migrate to the other end and drag the fluid with them to cause a slip velocity which propels the motor. ${ }^{9}$ However, macroscale catalytic motors are driven by recoiling oxygen gas bubbles as reported by Whitesides et al. ${ }^{3}$ One should therefore be curious if there is clear evidence for oxygen bubbles propelling the micro- or nanomotors by simple recoiling.

By using rolled-up nanotech, catalysts like $\mathrm{Ag}$ or Pt can be simply integrated into the inner wall of micro/nanotubes as shown in Fig. 3a. An optical image of a rolled-up Ti/Fe/Au/ $\mathrm{Ag}$ microtube is given in Fig. 3b. The metal Ti is used as the prestressed layer, and $\mathrm{Fe}$ is responsible for the magnetic control. $\mathrm{Au}$ is deposited in between the layers for better adhesion. The catalyst inside the tubes activates the $\mathrm{H}_{2} \mathrm{O}_{2}$ decomposition and the tube accumulates gas bubbles even if each individual bubble is too small to be resolved. The bubbles grow in size and are easily visualized by optical microscopy. As shown in Fig. 3c, an oxygen bubble tail is clearly observed and directly reveals the driving principle of our catalytic microtubular engines. Fig. 3d illustrates the driving mechanism. We identify a cyclic and asymmetric geometry change from a "single tube" into "a single tube with an attached bubble" and vice versa, which causes the motion of the microengines at low Reynolds number. The change from shape 1 (tube without bubble) to 2 (tube with bubble) causes a one step movement. Further expansion of the bubble (shape 3), which is still attached to the end of the tube, leads to an additional moving 
(a)

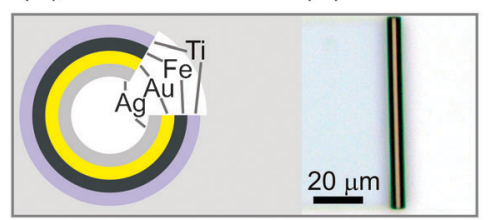

(d)

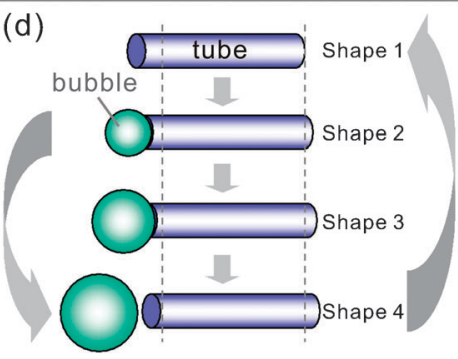

(c)

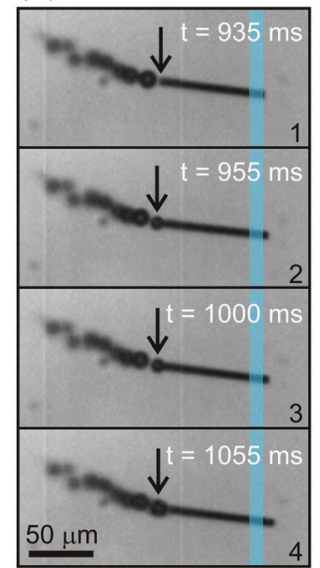

Fig. 3 (a) A sketch of the multi-material combination used in a microtubular engine, whose top view is show in (b) as an optical microscopy image. (c) Selected video frames of a self-propelled microrocket at different stages of the motion and (d) their corresponding schematic diagram. Reprinted with permission from ref. 18 .

step. Finally, the bubble grows and detaches from the tube end (shape 4), which generates a further "discrete" movement. Once detached, the tube retains the original shape (tube without bubble) to be ready for the next motion cycle. It should be noted that the expansion of bubbles (i.e. from shape 2 to 3 ) is sometimes omitted in particular if the bubble generation speed is very fast. Thus, shape 2 can directly transform into shape 4 and completes one cycle of motion.

In summary, when a rolled-up catalytic microtube is removed from the substrate, it is propelled by a recoiling mechanism produced by expelled microbubbles. The bubbles collect the gas inside the tube, expand, and then move to the larger opening, where they thrust the tube as they leave. The (small) change in diameter along the tube axis is expected to be linear for our rolled up nanomembranes. Thus, we can assume that the tubes are "cone-like" in shape with a small difference in diameter at the two tube openings. Due to simple hydrodynamic considerations the bubbles move to the larger opening and cause a pre-defined "directional motion/repulsion" once they are expelled from the microtube. The leading end of the moving tube is the smaller end. More extensive motion control of the tubes will be described in Section 6 .

\section{Speed of catalytic microengines}

The control and increase of the speed of catalytic micro- and nanoengines has been a subject of common interest since the first use of these nanostructures as self-propelled machines. ${ }^{4,5}$ The quest for more powerful catalytic engines has consequently led to high speed engines. The ability to increase the speed relies on the optimisation of different parameters such as the composition of the engines and the fuel solution.

There are several reasons to develop highly powered nanoengines. Among them, (i) high speed is desirable for rapid motility and transport in fluid and, (ii) if larger propulsion forces are achieved, heavier cargoes can eventually be transported over longer distances. Previously, the speed of

bimetallic nanomotors was observed to drop when immersed in microfluidic channels, which reduces their in vitro application potential. ${ }^{25}$ To avoid a dramatic decrease in nanomotor power, modifications and control over the fuel solution must be addressed. In the case of the rod-based catalytic nanomotors, some modifications of the "chassis" were proposed. ${ }^{26-28}$ First, the modification of the $\mathrm{Pt}$ end with carbon nanotubes was reported, which may increase the electrocatalytic surface for the decomposition of hydrogen peroxide. ${ }^{26}$ With the same goal in mind, the roughness of the catalytic surface was increased by mixing silica nanoparticles into the plating solution during the electrodeposition of $\mathrm{Pt}$ enhancing the peroxide breakdown and therefore the speed of the nanomotors. ${ }^{28}$ In addition, the incorporation of $\mathrm{Ag}-\mathrm{Au}$ alloys into the cathodic rod end replacing Au metal accelerated the nanomotors. ${ }^{27}$ Concerning the fuel solution, the speed of the nanomotors was observed to increase reaching a maximum value at different concentrations ranging from $2 \%$ to $15 \%$ depending on the nanomotor structure (i.e. bimetallic $\mathrm{Au}-\mathrm{Pt}$ rods $,{ }^{4} \mathrm{Au}-\mathrm{Pt} / \mathrm{CNT},{ }^{26}$ spherical nanomotor). ${ }^{29}$ Besides, the addition of hydrazine to the peroxide solution was observed to increase dramatically the speed of the nanomotors. ${ }^{26}$

Our catalytic microtubular jets are propelled by bubble recoil in contrast to the nanorod-based nanomotors which are self-propelled by other mechanisms (i.e. electrokinetic, diffusiophoretic, etc.). ${ }^{8-14}$ We carried out systematic studies, by varying both the structure of the microjet and the fuel solution, in order to increase the speed (by catalytic reaction power) while controlling the motion of the microjets. By optimizing the fuel composition we are able to control the speed of the microengines and, at the same time, the substitution of the inner Pt layer for an Au layer leads to a versatile platform for biofunctionalization.

\subsection{Fuel concentration}

Due to our particular propulsion mechanism, a deep understanding of the bubble growth and release mechanism is needed. Several steps are involved in the propulsion of our tubular structures. When the microtubes (containing a thin Pt layer inside of the rolled-up structure) are immersed into a hydrogen peroxide solution, microbubbles are generated in the cavity of the tube and released from the rear opening.

Because the Pt surface is hydrophobic, the metal layers refrain from mixing with the aqueous fuel solution. In order to achieve a complete wetting of the inside of the tube, we need to reduce the surface tension of the fuel solution. ${ }^{7}$ This reduction is achieved by dissolving surfactants into the solution which causes a huge effect on the motion of the tubular microengines. We firstly used common soap to stabilize the microbubbles, which led to improved propulsion and very fast engines. ${ }^{7}$ Moreover, because isopropanol has a low surface tension compared to peroxide solution, the addition of small amounts facilitated the visualization of the microengines motion since it locally reduced the high surface tension and decreased the bubble lifetime. By adding these two components to $15 \% \mathrm{H}_{2} \mathrm{O}_{2}$ solution, tubes with a diameter of $5.5 \mu \mathrm{m}$ and a length of $60 \mu \mathrm{m}$ moved at about $1.5 \mathrm{~mm} \mathrm{~s}^{-1}$ ( about 20 body lengths per second). 


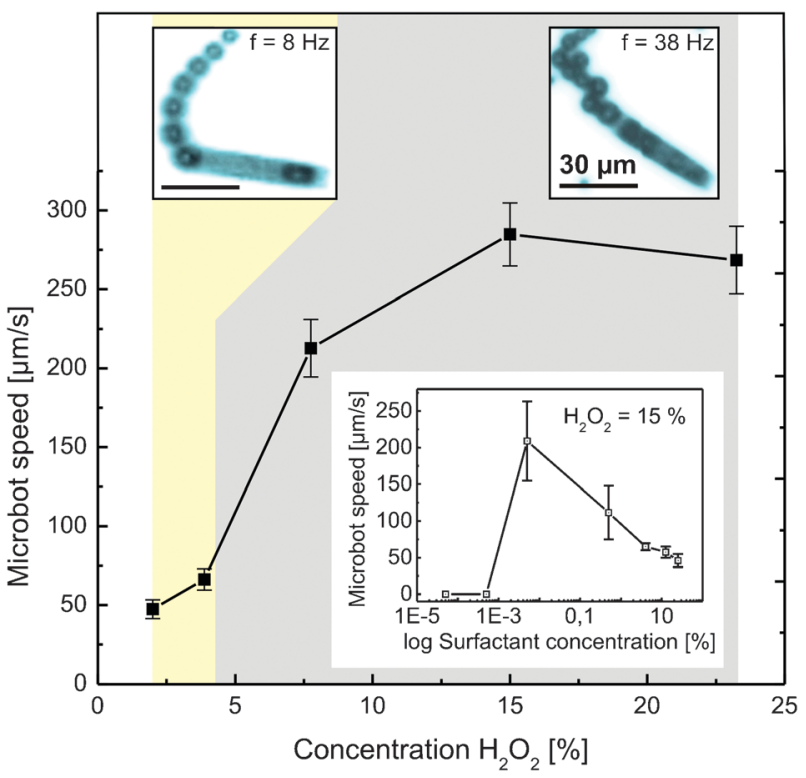

Fig. 4 Speed of microtubes with different concentrations of hydrogen peroxide containing a surfactant $(0.005 \mathrm{wt} \%$ benzalkonium chloride $)$ and $1 \mathrm{v} / \mathrm{v} \%$ of isopropanol. Inset shows the speed of microtubes with different concentrations of the surfactant $\left(5 \times 10^{-5}\right.$ to $\left.20 \%\right)$ at $15 \mathrm{wt} \%$ of $\mathrm{H}_{2} \mathrm{O}_{2}$. Reprinted with permission from ref. 16 .

Further studies with benzalkonium chloride demonstrated that a surfactant concentration beyond a certain threshold is required to activate the motion of the microengines (see inset of Fig. 4). ${ }^{16}$ Nonetheless, the speed is reduced by further increasing the surfactant concentration since above a certain point (critical micelle concentration, $\mathrm{cmc}$ ) the surface tension becomes constant and micelles may occupy the chemically active sites of $\mathrm{Pt}$, which in turn reduces the catalytic area of the microengine (see inset of Fig. 4). A similar effect was observed for Wang's catalytic tubular microengines, where Triton $\mathrm{X}-100$ influenced the motion of their tubular structures and therefore only a very low concentration of the surfactant was used for the propulsion. ${ }^{30}$

The catalytic surface of Pt contains nucleation points (i.e. defects, nanocavities) which induce the decomposition of $\mathrm{H}_{2} \mathrm{O}_{2}$ into water and molecular di-oxygen. These oxygen bubbles grow and expand into the cavity of the tube, before moving (migrating) towards the larger opening, where the pressure is lower. Finally, the recoil of the bubbles thrusts the microtubes in the opposite direction. In summary, the propulsion mechanism consists of four stages: (1) nucleation, (2) growth, (3) migration and (4) release of the bubbles that were collected inside of the microtube. It was observed that both migration and release stages induce fluid pumping, which have influence on the propulsion of the microjets. Indeed, the microjet engines propel in "moving steps" caused by the discrete bubble release. Therefore, the size and frequency of the released bubbles are important parameters for the motion.

The size and frequency of the bubbles can be controlled by changing the concentration of the hydrogen peroxide. It was observed that at higher fuel concentrations, the bubble frequency is significantly increased and at the same time the bubble radius reduces. It was found that for small bubbles, the average moving steps are almost equal to each bubble radius. However, when the radius of the released bubble increases, the moving steps become larger than the bubble radius. Therefore, when larger bubbles are generated $(>7 \mu \mathrm{m})$, longer moving steps are observed, which can be attributed to higher propulsive forces. ${ }^{7}$ A similar effect was recently reported by other catalytic tubular microengines of Wang's group. More specifically, they observed that a reduction in the bubble size leads to shorter moving steps and therefore less speed. ${ }^{30}$

Interestingly, by studying these two parameters of the bubbles (i.e. radius and frequency), we found that the velocity of the microengines is almost equal to the product of them. Consequently, we look for the optimum fuel composition to acquire a maximum bubble frequency and size. In our optimization studies of the fuel concentration, keeping the concentration of the surfactant constant, we observed how a low frequency of bubbles produces a low speed (Fig. 4.). But the speed increases as the bubble frequency inside the tube increases. This can be clearly seen in the insets of Fig. 4, where the maximum speed for the controlled fuel was found to be $38 \mathrm{~Hz}$. This maximum frequency also corresponds to the maximum speed $\left(275 \mu \mathrm{m} \mathrm{s}^{-1}\right)$ achieved for the selected fuel solution (i.e. $15 \% \mathrm{H}_{2} \mathrm{O}_{2}$ ). ${ }^{16}$ Because the space inside the tube is limited, there is a maximum frequency of bubbles which can be generated. By plotting the speed over the concentration of peroxide, a typical Langmuir adsorption isotherm is obtained, which relates the adsorption of molecules on a solid surface to the concentration of the medium. ${ }^{31}$ The maximum speed might be then limited by the surface area of the Pt catalyst layer and the turnover rate of the catalyst at high analyte concentrations.

\subsection{Types of catalysts}

We recently designed biocatalytic microengines which propel more efficiently than the Pt based microengines. ${ }^{32}$ In this hybrid configuration, we functionalized a thin $\mathrm{Au}$ layer with self-assembled-monolayers (SAM) where the catalase enzymes were covalently bonded. Catalase can decompose millions of hydrogen peroxide molecules every second into water and molecular oxygen which propels the microengine. These microengines are propelled at an average speed of about $226 \mu \mathrm{m} \mathrm{s}^{-1}$ when immersed in $1.5 \mathrm{wt} \%$ of $\mathrm{H}_{2} \mathrm{O}_{2}$ corresponding to about 10 body lengths per second compared with 1 body length per second for the $\mathrm{Pt}$ based microengines at that concentration of fuel. ${ }^{7,32}$ Therefore, we succeed in the fabrication of more efficient microjets while significantly reducing the toxicity of the fuel (from $15 \%$ to $1.5 \%$ of $\mathrm{H}_{2} \mathrm{O}_{2}$ to achieve the same speed).

\subsection{Speed in microchannels}

The only work on the use of catalytic nanomotors in microfluidic channels reported that their speed dramatically decreased about four times. ${ }^{25}$ In Fig. 5, we demonstrate that the microtubular engines are able to move against flows up to $600 \mu \mathrm{m} \mathrm{s}^{-1}$. Moreover, when the velocity of the fluid flow is zero, the microtubular engines acquire velocities up to nearly $300 \mu \mathrm{m} \mathrm{s}^{-1}$, which is in the same range as that for those immersed in free solution. ${ }^{7}$ The speed of the microengines is 
(a)

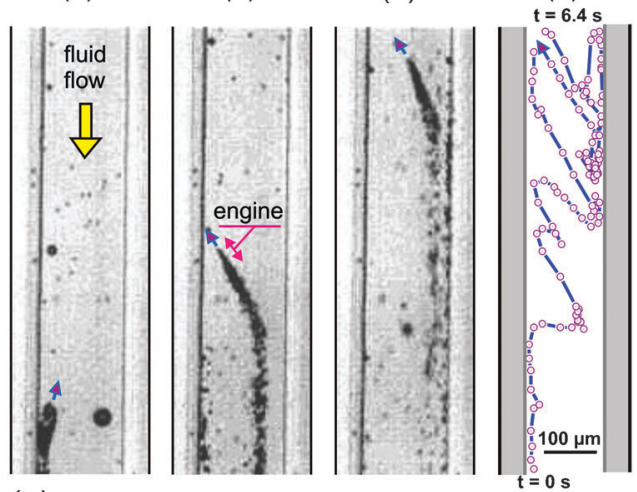

(e)

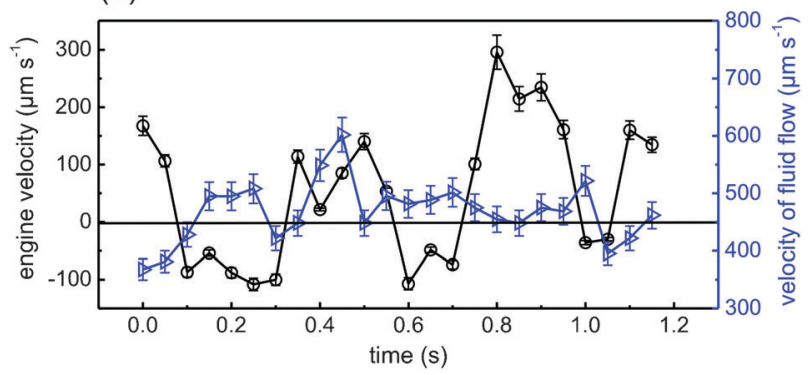

Fig. 5 A microtubular engine moving in a microchannel at (a) $0 \mathrm{~s}$, (b) $3 \mathrm{~s}$, and (c) $6.4 \mathrm{~s}$. (d) Its trajectory. (e) Velocities of the engines and fluidic flow as a function of time.

not affected by the absorption of peroxide into the PDMS microchannels since the flow maintains the supply of fuel to the microengines. We observed that the microengines tend to move towards the walls of the microchannels (as shown in Fig. 5d) where the laminar flow is slower. In contrast, when the engines are in the center of the channel, they are pushed back (negative velocity in plot Fig. 5e), coming forward again when they find lower resistance against the flow at the channel edge.

\section{Cargo transportation and assembly}

The transport of cargo along predefined tracks is essential for many life processes. Excellent examples are motor proteins which can sense, pick up and deliver intracellular cargoes to specific targets. ${ }^{33,34}$ For such reasons, these magnificent workhorses (dynein, kinesin and myosin) have motivated scientists to fabricate synthetic nanomotors with the ability to efficiently and precisely load-transport-deliver cargoes at well-defined sites. ${ }^{11,35}$

The first report of a nonbiological nanomotor able to transport cargo was presented by Sen's group, which consisted of $\mathrm{Pt}-\mathrm{Au}$ nanomotors transporting polystyrene microspheres. ${ }^{36}$ The electrodeposition of magnetic Ni segments into the nanomotors allowed the remote control of the motion of the doublet nanorod-cargo. In order to load the microparticles, the authors added extra functionalities to the Au segment of the nanorod, i.e. negative Ppy that electrostatically interacts with positively charged PS-amidine microparticles and attaches them to the nanomotor. A more specific biotinstreptavidin interaction was used as the second modification platform where the nanorod was modified with SAM of biotin-terminated disulfide binding PS-Streptavidin cargo.

Very recently, Sen's group solved another nanomotor limitation to release cargo by describing two routes to drop-off the cargo based on the dissolution of silver and a photocleavable bifunctional linker (PCL) triggered by UV light. ${ }^{37}$ However, the time required for drop-off was very long and their nanomotors could only bind specifically functionalized cargoes. Wang's group demonstrated directed motion of nanomotors and the transport of cargo within microchannel networks. ${ }^{25}$ Furthermore, by integrating a magnetic segment into their Pt-CNT-Au nanorod they succeeded in externally controlling the motion of their nanomotors as well as loading spherical magnetic cargo. The same group performed an electrodeposition method to fabricate catalytic tubular microengines containing a Ni layer that allows the guidance of the tubular structures as well as the pick up of magnetic cargoes. ${ }^{30}$ However, the release of the magnetic particle was not reported. Despite these excellent results, two drawbacks are identified which hinders the use of micro-/nanoengines as nanotools for delivery of cargoes: (i) extra functionality needs to be given either to the nano-/micromachine or to the cargo (e.g. magnetic properties, chemical modification, electrostatic interaction), and (ii) previous reports could only load one particle at a time.

In order to overcome these limitations, we took advantage of the pumping mechanism to induce suction of suspended microparticles into the microtubular structures and load them (Fig. 6a and b). It was observed that for catalytically active microtubes (i.e. immersed in peroxide fuel), polystyrene microparticles located nearby the opening of the tubes can be either absorbed to the tube entrance (if the particles are large) or pumped through (if the particles are small). For large particles, this pumping mechanism is dynamic, i.e. once the particles block the fluid from getting inside, the pumping/ sucking ceases and Brownian diffusion causes the particles to slightly move away from the opening so that pumping commences in a cyclic manner.

The engines can load and transport a large number of microparticles, implying a high propulsion power, which we calculate to be around $3.77 \mathrm{pN}$ for transporting one single $5 \mu \mathrm{m}$ diameter polystyrene particle. Accordingly, and following Stoke's law (Fig. 6c), there is a decrease in speed due to the increase of the drag force, when the number of transported microparticles is increased from 1 to 60 .

Because of the simple pumping mechanism to pick up the microobjects, no chemical modification of the microtube was needed. The external magnetic control allows us to selectively load specific microobjects and to transport them along predefined paths. The motion control was neither influenced by the size of the cargo nor the material transported. This observation was corroborated by transporting magnetic plates consisting of $\mathrm{Ti} / \mathrm{Fe} / \mathrm{Pt}$ films floating at the fluid/air interface. We used these square-patterned plates to demonstrate directed assembly, i.e. collecting and changing the configuration of the plates by applying deliberate changes in the magnetic field direction. In all cases, the delivery was accomplished by a very easy method compared to previous techniques: we quickly rotated the poles of the magnet at a speed faster than one rev s ${ }^{-1}$ 
(a)

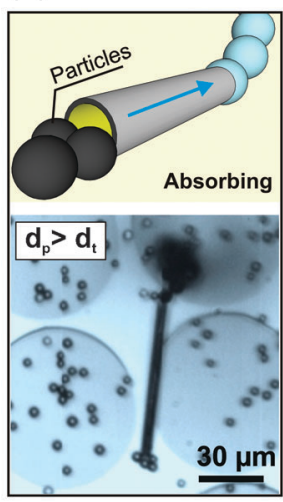

(c)

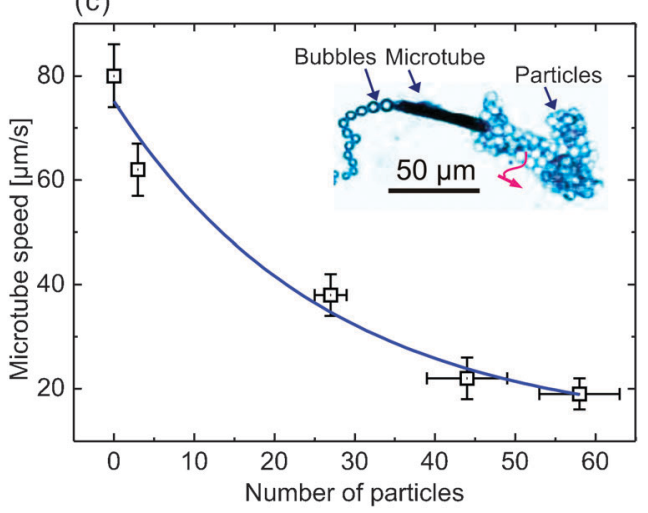

Fig. 6 Schematic diagrams (top) and optical images (bottom) of pumping effect with (a) big and (b) small particles. (c) Plot depicts the speed of microbots to number of loaded microparticles. The inset shows the transport of polystyrene microparticles of $5 \mu \mathrm{m}$ diameter. An optical microscope image of a microbot loading and transporting 58 particles. Reprinted with permission from ref. 16.

and observed that the particles immediately detach from the microtube and can be delivered at the desired position.

\section{Motion control and dynamic interaction}

As required for potential applications in drug delivery and nanoscale patterning, catalytic micro- and nanomotors need precise direction and speed control. ${ }^{15}$ In addition, individual instead of collective control of single machines is strictly required but still challenging to achieve. ${ }^{14}$ Wang et al. have recently developed different approaches to control the motion of catalytic nanomotors including magnetic guidance, thermally driven acceleration, electrochemical switching, and chemical stimuli. ${ }^{15}$ In the Penn State group, dynamic interactions between the motors are demonstrated at both individual and collective levels. ${ }^{13,38}$ In the latter case, the motion control is inspired by nature relying on chemotaxis and phototaxis. Furthermore, it was found that the motion of individual nanomotors may be also influenced by the chemical gradient generated by neighboring motors. ${ }^{38}$ In other words, control of motion can be also used as a sensing principle. Wang et al. have presented motion-based detection of silver traces. ${ }^{39}$ The speed of catalytic nanomotors is dramatically accelerated in the presence of silver ions and a better understanding will

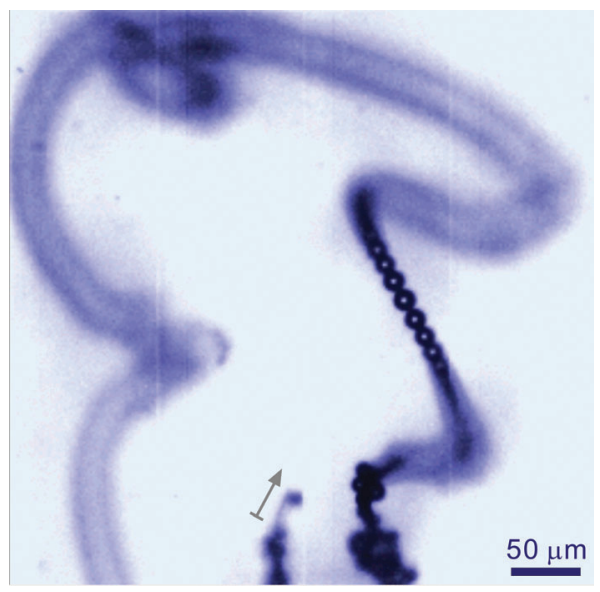

Fig. 7 An optical image of a long bubble tail illustrating the trajectory of one rolled-up tubular microengine (marked by the arrow).

further stimulate and promote the concept of motion-based sensing. ${ }^{40}$

Rolled-up catalytic microtubular engines collect oxygen gas, which is expelled as bubbles through one end. The bubbles can be stabilized by surfactants for visualization of the trajectories. ${ }^{7}$ One typical optical image (Fig. 7) shows a long bubble tail to illustrate the trajectory of one engine (highlighted by arrow). The trajectory is three dimensional which is well-monitored by the focused and defocused parts of the bubble tail, allowing us to easily track the position of the microtube engine. The geometries of the trajectories are given by the size and shape of the rolled-up microtubes as well as the catalytic machine power. Several interesting questions arise: (1) how stable are the bubbles? (2) how does the surfactant influence the motion? (3) can the formed bubbles help or speed up the motion? (4) why do the trajectories change dramatically? Is it only due to the geometry or size of our engines? Maybe fluid dynamics also affect the trajectories. The answers would lead to a comprehensive picture of the bubble-recoiling mechanism at the micro- and nanoscale.

Previously, methods have been developed to temporally and spatially manipulate the speed of catalytic motors. ${ }^{15}$ In the following, the catalytic engines are localised to a fixed position by a rotating magnetic field. Furthermore, we demonstrate that a glass capillary can fish individual tubular microengines out of the fluid due to capillary forces.

\subsection{Dynamic magnetic control}

As we discussed in Section 5, a static magnetic field can direct or guide the motion of our engines. Together with the pumping mechanism, the engines can load, transport, and release cargos at well-defined positions. One problem is, however, that these engines move constantly and the catalytic reaction cannot be stopped on demand. Dynamic magnetic control, i.e. magnetic control under an external rotating magnetic field is an attempt to localize moving engines. ${ }^{7}$ Microengines moving autonomously in straight lines at speed $v$ start to move on circular trajectories with radius $r$ when a rotating magnetic field is applied with a constant frequency $\left(f_{B}\right)$ as shown in Fig. 8a. When the linear speed is kept 

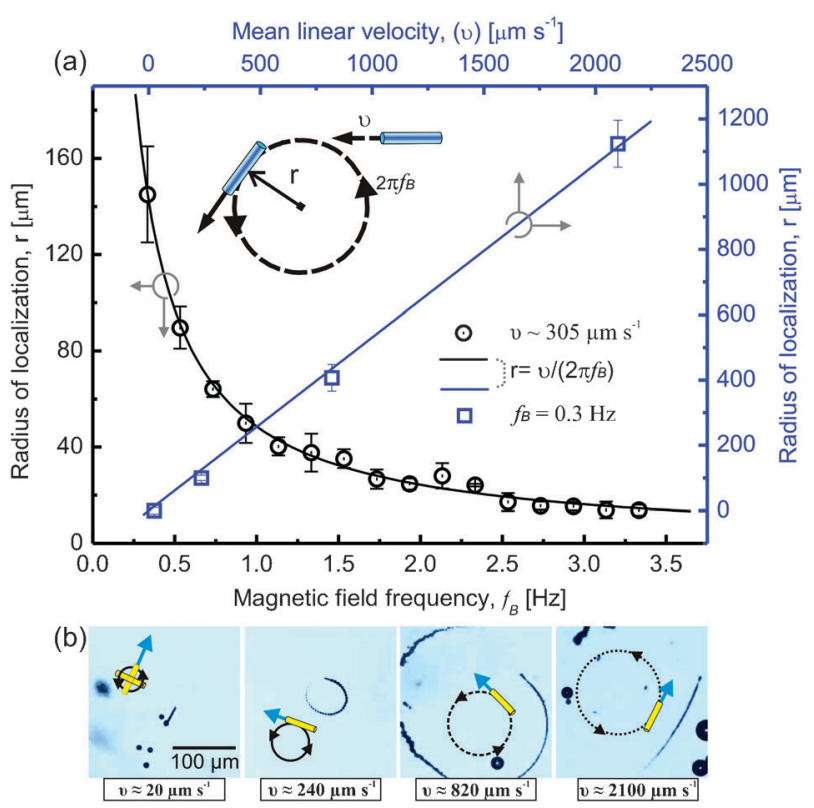

Fig. 8 Magnetic control of microjets, which self-propel originally in straight trajectories. (a) Localization radius as a function of rotating magnetic field frequency at a constant linear velocity (black circles) and dependence of localization radius on the mean linear velocity under a constant rotating magnetic field (blue squares). The solid lines are calculations. (b) Experimental video frames of a moving microjet at different speeds. Their frames correspond to the data points (blue squares) in (a). Yellow drawings with attached blue arrows represent moving microjets. Black circles with arrows schematically illustrate the applied rotating magnetic field. Reprinted with permission from ref. 7 .

constant, the localization radius decreases with increasing the rotating frequency. Vice versa, at a constant frequency, the radius is proportional to the linear speed $\left(r=\mathrm{v} /\left(2 \pi f_{B}\right)\right)$. When the speed is very low $\left(\sim 20 \mu \mathrm{m} \mathrm{s}^{-1}\right)$, the rotating behavior of the engine is different from that at higher speeds as shown in Fig. 8b. The slow engine rotates around it's very own center, while the faster engines travel on big circles with constant velocities. Interestingly, at higher magnetic field frequencies the microjets start to hit their own tails (i.e. bubbles), which causes fluctuations in their linear and angular velocities. Hitting their own tails means that the circular motions take place in one plane and thus magnetic control seems very promising for future applications.

Dynamic magnetic control may stimulate new ways of controlling catalytic micro- and nanoscale motors. For example, microwaves, surface acoustic waves, etc. can dynamically change the trajectories without modifying speed and/or fuel concentrations. Previous motion control was based on the modification of the environment ${ }^{15}$ which could affect or kill species "living" together with the artificial engines. Dynamic control is expected to help conducting missions, e.g. drug delivery or nanosurgery, without any detrimental influence. ${ }^{14}$

\subsection{Catalytic micro "water striders" and their control}

Water striders in nature walk on the water surface and land by the meniscus-climbing effect. ${ }^{41}$ Capillary forces are the key for their landing. Here, we employ rolled-up microtubes as catalytic micro "water striders" at the air-liquid interface of hydrogen peroxide $\left(\mathrm{H}_{2} \mathrm{O}_{2}\right)$ solution. ${ }^{42}$ Such micro-/nanotubes buoyed and self-propelled by oxygen bubbles move at the fuel surface by bubble recoiling, and easily attached to micro-objects by the meniscus-climbing effect, which is one of the main features found in the behaviour of water striders. ${ }^{41}$ The balance between capillary and drag forces determines assembly and disassembly of the microtubes, which thus exemplifies a dynamic self-assembly behaviour of micromachines on the micro- and nanoscale. Importantly, a glass capillary can simply fish or capture the engines, which enables interaction between the individual motors and human beings.

The basic principle is sketched in Fig. 9a, where catalytic microtubular engines firmly attached to bubbles can attract each other at an aqueous fuel surface and self-assemble into patterns due to the meniscus-climbing effect. The bubbles pull attached microtubes together and form self-assembled structures of microtubes. The microtubular engines are well aligned in certain directions and pile up in abundance (Fig. 9b). Attracting capillary forces induced by the meniscus-climbing effect can be studied by inserting a glass capillary into the surface to anchor the microengines. Fig. 9c shows a glass capillary inserted into the fuel and thus modifying the fluid surface. Several catalytic microengines, having moved around previously, rush spontaneously towards the capillary at the liquid surface, and representative images for three microengines are shown in Fig. $9 d-f$, which correspond to the tubes i-iii shown in Fig. 9c, respectively. As schematically shown in the inset of Fig. 9c, a generated bubble with a connected tube is pulled

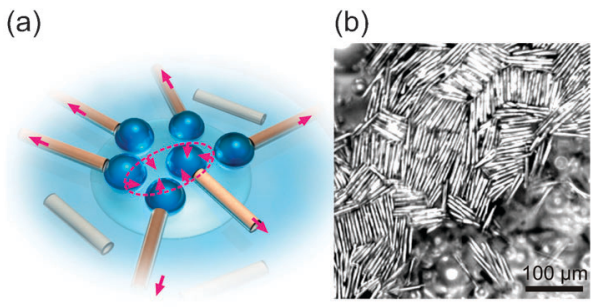

(c)

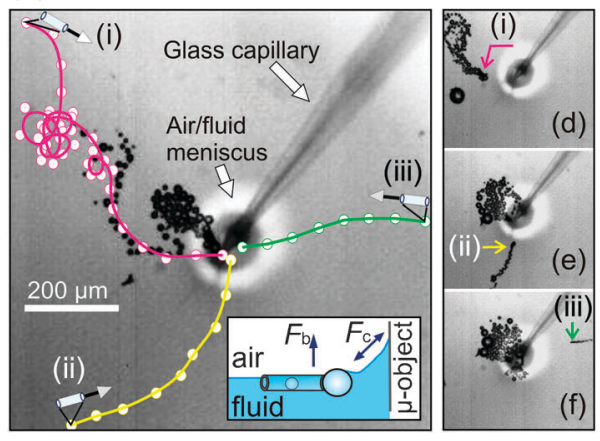

Fig. 9 (a) Schematic illustration of bubble-attached catalytic microtubular engines attracted to each others. The individual arrows represent the repulsion forces due to the bubble recoiling mechanism, while the arrows within the dashed circle denote the meniscus-climbing effect (attraction) initiated by capillary forces. (b) Optical image of a self-assembled microtube array, where $100 \mu \mathrm{l}$ of $30 \% \mathrm{H}_{2} \mathrm{O}_{2}$ fuel was added into $200 \mu \mathrm{l} \mathrm{PC}$. (c) The meniscus-climbing microtubes (i-iii) rushing towards a glass capillary. The lines and solid circles show the corresponding trajectories for each engine. (d-f) Selected video frames for the individual microtubes in (c). The arrows highlight the moving microtubes. Reprinted with permission from ref. 42 . 


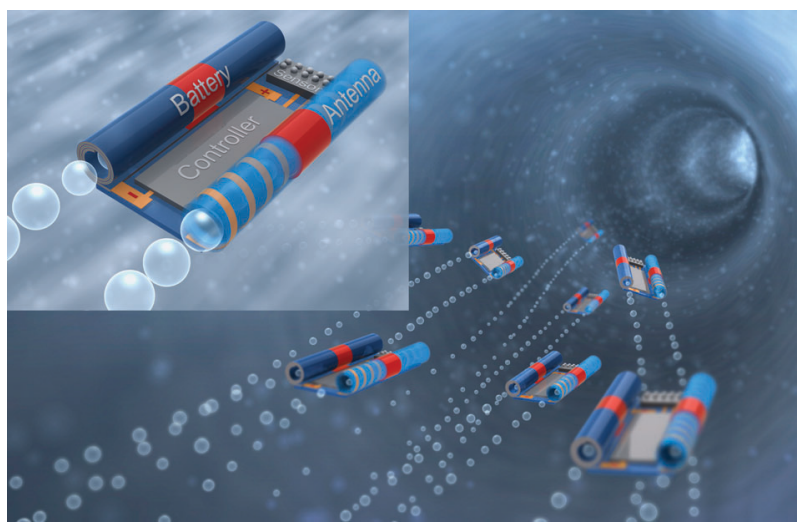

Fig. 10 Proposed sketch for smart catalytic microtubular engines. Components to fulfill tasks are marked in the inset. The main components are two oppositely rolled-up tubes, where the left one integrates an engine and a battery, and the right one acts as an antenna (for receiving external signal) as well as an engine. The IC controller is positioned and processed in between. The front end of the flat area is occupied by a sensor and a drug delivery component (marked by dots).

towards a micro-object (here glass capillary) by the capillary force $\left(F_{\mathrm{c}}\right)$, while the buoyancy force $\left(F_{\mathrm{b}}\right)$ from the bubbles lifts the tube to the fluid surface. In addition, when the capillary tip is removed from the liquid, the striders still stay at the air-liquid interface but move away randomly. In this way we can exactly place several catalytic microengines at certain positions at a certain requested time.

Our approach suggests that micro- or nanomotors can be collected and released by an external object, which is manipulated by a person. Further studies on the control of dynamic self-assembly will provide answers to the following questions. Can we introduce magnetic forces (instead of capillary forces) by integrating magnetic materials into the tubes to balance and manipulate the driving forces? Can we exploit dynamic self-assembly for patterning at the micro- and nanoscale? Can we realize dynamic self-assembly on demand, i.e. can the self-assembly pattern be varied upon an external signal?

\section{Summary and outlook}

In this article, we have reviewed the recent progress on catalytic microtubular engines fabricated by rolled-up nanotech on polymers. Comparison with other catalytic micro- and nanomotors is given throughout the text. Challenges and opportunities of such engines have been discussed and analyzed for future studies. Rolled-up nanotech on polymers can integrate almost any inorganic material into the engines and thus provide advanced functionalities such as power enhancement, cargo delivery, motion control, and dynamic assembly.

Over the last 6 years, catalytic micro- and nanomotors have attracted great attention and have been further developed to push the limits of tiny motors as suggested by Feynmann. ${ }^{43}$ However, there is still a long way for catalytic micro- and nanomotors to compete with nature's biomotors. ${ }^{17}$ The aim in the near future would be to produce more intelligent motors. ${ }^{13}$ How can these motors sense or detect the environment and react to these influences by their motion? Can they communicate with each other like nature's biomotors? One promising way would be the development of a highly integrative micro- or nanosystem which is composed of a catalytic power engine, an integrated circuit (IC) as the controller, a battery for powering the IC, an antenna for communication, a sensor to detect the environment, and a component to fulfill a mission e.g. drug delivery. Rolled-up nanotech has been rigorously developed over recent years and already offers several functional units such as engines, ${ }^{5,16,18}$ batteries, ${ }^{44,45}$ optical components, ${ }^{46}$ and sensors. ${ }^{47}$ One possible design of such a multifunctional engine is given in Fig. 10. All components can be constructed based on rolled-up micro- or nanotubes. As shown in the inset of Fig. 10, the main components are two oppositely rolled-up tubes, ${ }^{48}$ where the left one integrates an engine and a battery, and the right one acts as an antenna (for receiving external signal) as well as an engine. The IC controller is positioned and processed in between. One end of the flat area is occupied by a sensor and a drug delivery component. Such an engineered smart microtubular twin jet would be able to meet in parallel several requirements for a powerful and intelligent micromotor. We hope our concept motivates further exploration of rolled-up microengines as well as stimulates similar designs of other types of catalytic micro- and nanomotors.

\section{Acknowledgements}

We are grateful for fruitful discussions with all members in the Nanomembranes-ABC group, and experimental help by Ronny Engelhard and Cornelia Krien. This work was financially supported by the Grant from the Volkswagen Foundation (I/84 072). YFM also thanks the support from the Visiting Scholar Foundation of Key Laboratory of Opto-electronic Technology and Systems in Chongqing University.

\section{Notes and references}

1 From Wikipedia at http://en.wikipedia.org/wiki/Jet_engine.

2 E. M. Purcell, Am. J. Phys., 1977, 45, 3.

3 R. F. Ismagilov, A. Schwartz, N. Bowden and G. M. Whitesides, Angew. Chem., Int. Ed., 2002, 41, 652.

4 W. F. Paxton, K. C. Kistler, C. C. Olmeda, A. Sen, S. K. St. Angelo, Y. Cao, T. E. Mallouk, P. E. Lammert and V. H. Crespi, J. Am. Chem. Soc., 2004, 126, 13424-13431.

5 S. Fournier-Bidoz, A. C. Arsenault, I. Manners and G. C. Ozin, Chem. Commun., 2005, 441-443.

6 M. Pumera, Nanoscale, 2010, $2,1643$.

7 A. A. Solovev, Y. F. Mei, E. Bermudez Urena, G. Huang and O. G. Schmidt, Small, 2009, 5, 1688, and references therein.

8 W. F. Paxton, S. Sundararajan, T. E. Mallouk and A. Sen, Angew. Chem., Int. Ed., 2006, 45, 5420.

9 G. A. Ozin, I. Manners, S. Fournier-Bidoz and A. Arsenault, $A d v$. Mater., 2005, 17, 3011.

10 S. J. Ebbens and J. R. Howse, Soft Matter, 2010, 6, 726.

11 S. Sanchez and M. Pumera, Chem.-Asian J., 2009, 4, 1402.

12 T. Mirkovic, N. S. Zacharia, G. D. Scholes and G. A. Ozin, Small, 2010, 6, 159.

13 Y. Hong, D. Velegol, N. Chaturvedi and A. Sen, Phys. Chem. Chem. Phys., 2010, 12, 1423.

14 W. R. Browne and B. L. Feringa, Nat. Nanotechnol., 2006, 1, 25.

15 J. Wang and K. M. Manesh, Small, 2010, 6, 338.

16 A. A. Solovev, S. Sanchez, M. Pumera, Y. F. Mei and O. G. Schmidt, Adv. Funct. Mater., 2010, 20, 2430.

17 J. Wang, ACS Nano, 2009, 3, 4 
18 Y. F. Mei, G. S. Huang, A. A. Solovev, E. Bermudez Urena, I. Monch, F. Ding, T. Reindl, R. K. Y. Fu, P. K. Chu and O. G. Schmidt, Adv. Mater., 2008, 20, 4085.

19 K. M. Manesh, M. Cardona, R. Yuan, M. Clark, D. Kagan, S. Balasubramanian and J. Wang, ACS Nano, 2010, 4, 1799.

20 O. G. Schmidt and K. Eberl, Nature, 2001, 410, 168.

21 O. G. Schmidt, N. Schmarje, C. Deneke, C. Müller and N.-Y. Jin-Phillipp, Adv. Mater., 2001, 13, 756.

22 L. Zhang, J. J. Abbott, L. X. Dong, K. E. Peyer, B. E. Kratochvil, H. X. Zhang, C. Bergeles and B. J. Nelson, Nano Lett., 2009, 9 , 3663.

23 Y. F. Mei, D. J. Thurmer, F. Cavallo, S. Kiravittaya and O. G. Schmidt, Adv. Mater., 2007, 19, 2124.

24 C. Deneke, C. Müller, N. Y. Jin-Phillipp and O. G. Schmidt, Semicond. Sci. Technol., 2002, 17, 1278.

25 J. Burdick, R. Laocharoensuk, P. M. Wheat, J. D. Posner and J. Wang, J. Am. Chem. Soc., 2008, 130, 8164.

26 R. Laocharoensuk, J. Burdick and J. Wang, ACS Nano, 2008, 2, 1069.

27 U. K. Demirok, R. Laocharoensuk, K. M. Manesh and J. Wang, Angew. Chem., Int. Ed., 2008, 47, 9349.

28 N. S. Zacharia, Z. S. Sadeq and G. A. Ozin, Chem. Commun., 2009, 5856.

29 J. G. Gibbs and Y.-P. Zhao, Appl. Phys. Lett., 2009, 94, 163104.

30 K. M. Manesh, M. Cardona, R. Yuan, M. Clark, D. Kagan, S. Balasubramanian and J. Wang, ACS Nano, 2010, 4, 4.

31 Chemistry on the Solid-Water Interface, ed. W. Stumm, John Wiley and Sons, New York, NJ, 1993, ch. 4.

32 S. Sanchez, A. A. Solovev, Y. F. Mei and O. G. Schmidt, J. Am. Chem. Soc., 2010, 132, 13144.

33 A. Goel and V. Vogel, Nat. Nanotechnol., 2008, 3, 465-475.

34 M. G. L. V. den Heuvel and C. Dekker, Science, 2007, 317, 333.
35 M. Schliwa and G. Woehlke, Nature, 2003, 422, 759-765.

36 S. Sundararajan, P. E. Lammert, A. W. Zudans, V. H. Crespi and A. Sen, Nano Lett., 2008, 8, 1271.

37 S. Sundararajan, S. Sengupta, M. E. Ibele and A. Sen, Small, 2010 , 6, 1479.

38 Y. Wang, S.-t. Fei, Y.-M. Byun, P. E. Lammert, V. H. Crespi, A. Sen and T. E. Mallouk, J. Am. Chem. Soc., 2009, 131, 9926.

39 D. Kagan, P. Calvo-Marzal, S. Balasubramanian, S. Sattayasamitsathit, K. M. Menesh, G.-U. Flechsig and J. Wang, J. Am. Chem. Soc., 2009, 131, 12082.

$40 \mathrm{~J} . \mathrm{Wu}, \mathrm{S}$. Balasubramanian, D. Kagan, K. M. Manesh, S. Campuzano and J. Wang, Nat. Commun., 2010, 1, 36.

41 D. L. Hu and J. W. M. Bush, Nature, 2005, 437, 733.

42 A. A. Solovev, Y. F. Mei and O. G. Schmidt, Adv. Mater., 2010, 22, 4340 .

43 R. P. Feynmann, There's Plenty of Room at the Bottom: an Invitation to Enter a New World of Physicshttp://www.zyvex. com/nantech/Feynman.html.

44 H. X. Ji, Y. F. Mei and O. G. Schmidt, Chem. Commun., 2010, 46, 3881.

45 C. C. Bof-Bufon, J. D. Cojal Gonzalez, D. J. Thurmer, D. Grimm, M. Bauer and O. G. Schmidt, Nano Lett., 2010, 10, 2506.

46 V. A. Bolaños Quiñones, G. S. Huang, J. D. Plumhof, S. Kiravittaya, A. Rastelli, Y. F. Mei and O. G. Schmidt, Opt. Lett., 2009, 34, 2345.

47 G. S. Huang, V. A. Bolaños Quiñones, F. Ding, S. Kiravittaya, Y. F. Mei and O. G. Schmidt, ACS Nano, 2010, 4, 3123.

48 O. G. Schmidt, Ch. Deneke, S. Kiravittaya, R. Songmuang, H. Heidemeyer, Y. Nakamura, R. Zapf-Gottwick, C. Müller and N. Y. Jin-Phillipp, IEEE J. Sel. Top.Quantum Electron., 2002, 8, 1025 